\title{
ASSESSING THE EFFICIENCY OF JING-JIN-JI COLLABORATIVE DEVELOPMENT IN DIFFERENT LOW CARBON ECONOMY STRATEGIES AROUND XIONGAN WITH SD AND FRACTAL MODEL
}

\author{
XU, W.-B. ${ }^{1}-$ ZHANG, X.-H. ${ }^{1 *}-$ WANG, X.-S. ${ }^{1}-$ ZHANG, B.-A. ${ }^{2 *}$ \\ ${ }^{1}$ Institute of Environmental Economics, Tianjin Polytechnic University, Tianjin 300387, China \\ ${ }^{2}$ Hebei Environment Engineering University, Qinhuangdao City 006112, China \\ *Corresponding authors \\ e-mail:xuehua671231@163.com (X.-H.Zhang),15033529696@126.com (B.-A.Zhang)
}

(Received 23 $3^{\text {rd }}$ Apr 2019; accepted $4^{\text {th }}$ Jul 2019)

\begin{abstract}
The paper presents a new method to evaluate the impact of Xiongan's construction on the collaborative development of Jing-Jin-Ji (JJJ) Urban Agglomeration under different development strategies. Firstly, the SD model of each urban node of JJJ Urban Agglomeration is constructed by using parallel calibration system, and it is applied to simulate the development trend of target system. Then, the paper sets up a fractal model base on gravitational theorem which takes population, GDP and full carbon emissions as main factors to be evaluated with the Low Carbon Economic Linkage Strength. The evaluation results show that: (1) Xiongan as a radiation center can significantly promote the collaborative development of urban agglomeration; (2) On the basis of the existing trend, improving the low-carbon level of Xiongan alone has no significant effect on the low-carbon development, while the effect is significant when Beijing, Tianjin and Xiongan low-carbon levels are improved at the same time; (3) There will be a delay in the transmission of low-carbon economic development capacity from high to low gradient, but this delay does not affect the radiation effect of high gradient cities on low gradient cities, nor does it affect the coordinated development of urban agglomerations.
\end{abstract}

Keywords: low-carbon economic strategy, fractal dimension, JJJ Urban Agglomeration, scenario analysis

\section{Introduction}

The Nineteenth Congress of the Communist Party of China pointed out that the main contradiction in the current Chinese society is the contradiction between the people's pursuit of a better life and the insufficient balance of development (Pan et al., 2009). The contradiction of imbalanced development is particularly prominent in Jing-Jin-Ji (JJJ) Urban Agglomeration.

The JJJ Urban Agglomeration includes Beijing, Tianjin, Xiongan New District, Shijiazhuang, Tangshan, Baoding, Langfang, Handan, Zhangjiakou, Chengde, Qinhuangdao, Cangzhou, Xingtai and Hengshui. The total area of the JJJ Urban Agglomeration is $216,000 \mathrm{~km}^{2}$. It is dominated by plain landforms and belongs to temperate monsoon climate and semi-humid area. As one of China's important economic growth pole, the JJJ Urban Agglomeration is confronted with challenges to achieve sustainable development. The main challenge is facing two significant gradient differences ( $\mathrm{Li}$ et al., 2013). One is the social-economic gradient, the other is the resource-environment gradient. Beijing and Tianjin show the high gradient of social economy, while the node cities in Hebei show the high gradient of resources and environment. These two kinds of gradient differences make JJJ Urban Agglomeration become the most acute contradiction area in China. 
In this context, JJJ Urban Agglomeration is expected to become a model of urban agglomeration collaborative development and play a leading role in China's low-carbon development. As a newly rising radiation center of JJJ Urban Agglomeration, Xiongan has attracted much attention at home and aboard, which includes the influence of Xiongan's geographical location and different development modes on JJJ Urban Agglomeration.

The study of urban agglomeration has a long history, including both micro-scale and macro-scale research. The research involves in exploring the driving forces for the development of urban agglomerations with different focusing modes by comparing the frontiers of total factor productivity among urban agglomeration (Jia et al., 2018), sharing, matching, and learning mechanisms within urban agglomeration (Duranton et al., 2003), the dynamic mechanism of the growth of cities and urban agglomerations (Cao et al., 2012; White et al., 1994), urban location and boundary delimitation (Tannier et al., 2011), and political policies and their impact on the development of urban agglomerations (Jefferson et al., 2013). Fractal is the most commonly used method in urban agglomerations' research. Factual theory and method have been applied to analyze morphological boundaries of urban agglomerations and characterize boundary shapes (Tannier et al., 2013), and discovery the difference between planned patterns and less planned ones in morphological features of urban agglomerations (Frankhauser, 1998).

With the increasingly prominent environmental problems, it has become a worldwide concern for economists, as is witnessed by many theories and methods which aims at driving the economy towards a sustainable economy (Camagni et al., 1998). The research on urban agglomerations are gradually expanding from the economic system to environmental-economic complex system, such as the heat island effect of high-density urban agglomerations (Zhou et al., 2018), the driving force of low-carbon development (Kang et al., 2018), the evaluation of sustainable development level of urban agglomerations with multiple resource and environmental indicators (Yu et al., 2010).

Different environments co-exist in a city including the natural, the economic production and the social activation. Each of them generates positive and negative externalities for the city, since each of them represents 'use advantages' and 'use costs' for a city. For social activation, these externalities are manifested in energy consumption and food consumption (biomass resource consumption) and waste discharge in the daily life of clothing, food, shelter and transportation. For economic production, these externalities are finally measured in terms of resource and energy input, positive output (GDP) and negative output (waste emissions). While all the supply of natural resources and degradation of environmental pollutants are related to urban land area. Therefore city's quality of life and environmental concerns undermine all advantages associated with agglomeration economies.

SD model is a useful tool to analyze and forecast urban or city environmental economic system, which is widely used in the system simulation of homogeneous regions and the systematic analysis (Wu et al., 2018), as well as the study of expansion to the periphery of the target city (Rich et al., 2016; Yu et al., 2018). In the research of scenario analysis and strategy selection, the socio-economic plan of the target system is always one of the most important bases for scenario design, especially in carbon emission reduction ( $\mathrm{Du}$ et al., 2018) and effective utilization of natural resources (Zhang et al., 2008). However, due to the differences of natural conditions and development patterns in heterogeneous regions, the study of urban agglomerations 
focusing on these regions is very rare at present. Furthermore, there is another bottleneck problem, that is, how to normalize multiple resources and environmental factors in SD model.

There is no doubt that the dynamics of cities can be best captured by looking at them as complex systems governed by freedom, interacting on different space and time scales in a non-linear fashion (Albeverio et al., 2008). The evolution of cities often depends on the interaction of internal factors (such as decision-making of institutions and individuals) and external factors (radiation between cities and social progress). Therefore, the combination of SD model based on urban metabolic analysis and inputoutput flow can better solve the obvious heterogeneity in urban agglomeration system simulation and improve the integrity evaluation of urban agglomeration (Elliot et al., 2018).

In addition, the research on low carbon is developing rapidly from the initial concern of carbon emissions accounting of energy consumption to potential carbon environmental capacity of waste emissions and biomass resources consumption. The potential carbon environmental capacity occupancy of waste emissions involves both production (Tan et al., 2018) and living areas (Sun et al., 2018). While the potential carbon environmental capacity occupancy of biomass resources mainly involves living areas, whose theoretical basis is that the more land resources occupied by human demand for food, the less land resources used for the growth of high carbon-fixing vegetation (Timothy et al., 2018). The above research lays a foundation for the proposal of carbon accounting and the solution of the problem of resource and environment normalization in SD model.

Based on the analysis of system dynamics, this paper applies fractal theory and method to analyze the influence of Xiongan New District on the coordinated development of JJJ Urban Agglomeration from the perspective of low-carbon economic analysis, aiming to analyze the Xiongan New District to the promotion of low-carbon development of JJJ urban agglomeration. And this research provides a scientific basis for relevant departments to make decisions.

\section{Materials and methods}

Each node city in JJJ Urban Agglomeration has distinct heterogeneity. There are not only huge economic gradients, but also huge resource and environment gradients. In order to objectively evaluate the role of Xiongan in the low-carbon coordinated development of JJJ Urban Agglomeration and the impact of different strategies on the role, this paper proposed an integrated method which combined the system dynamics with the fractal analysis. The proposed method integrated progress and the paper following structure as: (1) analyzing JJJ Urban Agglomeration' system and identifying the Key Factors (KFs) that affect the low-carbon development; (2) setting up the framework of full carbon emission calculation cored in KFs; (3) establishing a SD model of social-economic-environmental complex system for each city in JJJ Urban Agglomeration to predict the development tendency cored in KFs; (4) based on the gravity theory, setting up the fractal model of low-carbon economic linkages strength of urban agglomerations cored in KFs; (5) designing different low-carbon scenario strategies according to system development trend prediction and relevant plan and evaluating the efficiency of JJJ collaborative development under different low carbon economy strategies around Xiongan. 
The material of this paper comes from multi-source data of each node in JJJ Urban Agglomeration, including data of social and economic development, data of resource consumption and data of pollution emission.

\section{System analysis and KFs identification}

\section{Analysis of heterogeneity of JJJ}

Land is the carrier of human activities in production and life, and the carrying capacity of natural resources and environment are closely related to urban land area. Thus in this paper, the heterogeneity of JJJ Urban Agglomeration is explored based on the analysis of the density of GDP, the density of population, the intensity of biological resources consumption, the energy consumption intensity, and the intensity of pollutant emission. The degrees of various indicators above are shown in Figure 1.
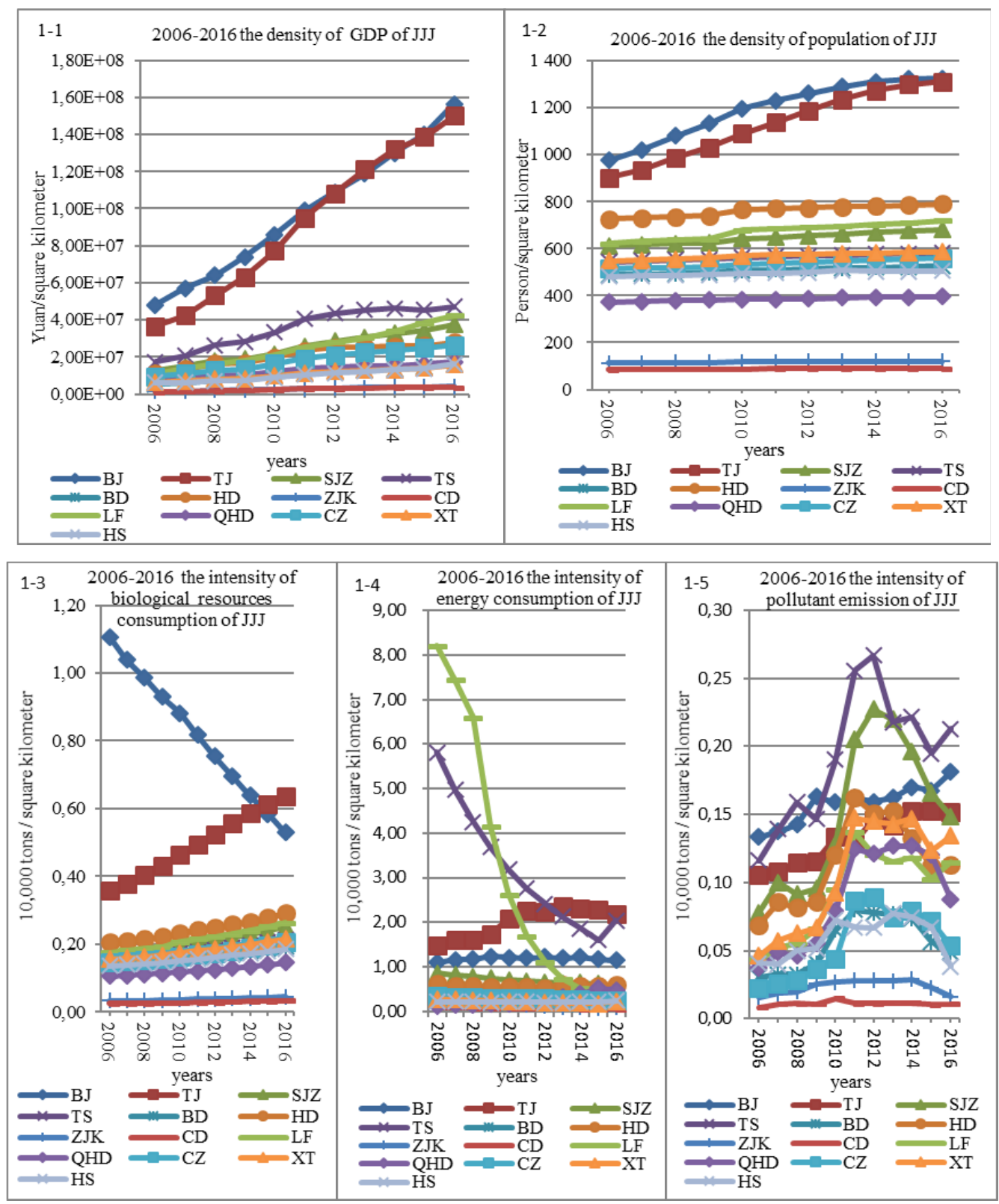

Figure 1. The level or intensity of social economy and resource environment of JJJ 
It can be seen from Figure 1-1 that Beijing and Tianjin are in a high gradient from the perspective of GDP density, while the other node cities of JJJ Urban Agglomeration are in low gradient, and there is a significant gradient difference in Beijing and Tianjin.

It can be seen from Figure 1-2 that Beijing and Tianjin are still in a high gradient in terms of population density, while Langfang, Shijiazhuang, Xingtai, Baoding, Tangshan, Zhangzhou, Hengshui and Qinhuangdao are in the middle gradient group, and meanwhile Zhangjiakou and Chengde are at a low level. The gradient difference between the above high, medium and low gradients is also very obvious.

It can be seen from Figure 1-3 that Beijing and Tianjin are high gradient from the perspective of biological resources consumption intensity (corresponding to the biomass resources output per unit area), while Shijiazhuang, Tangshan, Baoding, Handan, Langfang, Qinhuangdao, Cangzhou, Xingtai and Hengshui are in the middle gradient group, and meanwhile Zhangjiakou and Chengde are at a low level. Among them, there is a huge gradient difference between high gradient and middle gradient.

It can be seen from Figure 1-4 that Langfang gradually decreases from a high gradient to a low gradient in terms of energy consumption intensity, while Tangshan gradually reduced from the second highest gradient to the same level in Beijing. From the distribution of gradient after 2012, Tianjin is always in the high gradient, followed by Tangshan and Beijing. The above three cities together constitute a high gradient of energy consumption, while the rest has much less gradient of energy consumption.

It can be seen from Figure 1-5 that Tangshan is always at the highest gradient in terms of pollutant emission, while Shijiazhuang has a large fluctuation, ranging from middle gradient to high gradient. And in recent years, Shijiazhuang has a drop trend. Beijing and Tianjin are relatively stable. At the same time, Cangzhou, Hengshui and Baoding are in the middle-low gradient, while Chengde and Zhangjiakou are in low gradient.

As can be seen from the above analysis, the conclusions can be drawn that the heterogeneity of social economic, resource and environmental of the cities and towns of the JJJ Urban Agglomeration are extremely clear. Therefore, system development predictions and kinetic models for such urban agglomerations need to be built under the same input and output dynamics framework.

\section{Identifying the KFs of JJJ based on urban metabolism}

The main input of the urban system is all kinds of resources that can be provided by nature (including water environmental capacity, atmospheric environmental capacity, carbon storage capacity generated by forest resources, and various types of energy, etc.), and some of these resources are consumed through human economic activities. It is converted into products and waste, and the other part is consumed or converted into waste through human production activities. With the increase of waste discharge, the demand for environmental capacity increases accordingly. In order to control waste emissions within naturally available environmental capacity, it is necessary to treat waste generated during human production and life to reduce its emissions. Meanwhile, the process also consumes resources and energy, resulting in a carbon footprint. Therefore, waste emissions should be included in the potential carbon environmental capacity account. In addition, the biomass resources consumed in human life are rising, and the land available for growing high carbonaceous plants is declining. Therefore, biomass resource consumption should be included in the potential carbon environmental capacity occupancy account. The products formed during the production process 
(measured in terms of GDP) enter the next circulation area, or as an input enter the next activity, which is either consumed in the activity or a new product is formed. Then, they go to the next activity or become a waste into the environment. The analysis of the metabolic process of the above cities shows that human consumption of biomass resources and waste emissions (including $\mathrm{CO}_{2}$ and other emissions in the process of energy consumption, wastewater emissions and solid waste emissions) are integrated into the carbon emission accounting system, which can fully reflect the impact of urban production and living processes on the environment.

Therefore, the KFs affecting the low-carbon economy can be identified as follows: population, GDP, waste, energy and biomass resources. Of course, the population as one of the KFs is a subsystem, which is determined by the density of urban employment, the proportion of the population receiving higher education, the natural growth rate and the urbanization rate. GDP, one of the KFs, can also constitute a subsystem, which is composed of per capita GDP, economic density and per capita investment in fixed assets. Waste gas, wastewater and solid waste can form a waste discharge subsystem, which produces the amount of behavior, the amount of treatment (or treatment rate), and the impact of social and economic activities related to it. Energy consumption will also lead to the corresponding carbon emissions. As this part of the emissions is relatively large and has been the concern for a long time, it also becomes a subsystem. The consumption of biomass resources corresponds to the land occupation. The more biomass resources consumed by human life, the more land occupied for the production of these biomass resources, and the less land used for carbon sequestration (planting high carbon sequestration plants). Therefore, the consumption of biomass resources is also a KF. Accordingly, urban greening plays a role of beautifying life and absorbing carbon, so it is also one of the KFs.

\section{Construction the calculation framework of "full carbon emission"}

The "full carbon emission" account is set up cored in KFs based on the energy analysis theory and the energy conversion law (Zhang et al., 2015). The account consists of two sub-accounts, which are direct carbon emissions caused by energy consumption and potential carbon consumption caused by waste carbon emissions as well as biomass resources. Different types of substances are normalized by converting into $\mathrm{CO}_{2}$ emissions to characterize the impact of the emissions on the green low-carbon development of urban agglomerations. Considering the contribution of different subitems to direct and potential emissions account as well as the availability of data, the two sub-accounts are divided into several sub-sub accounts as shown in Figure 2.

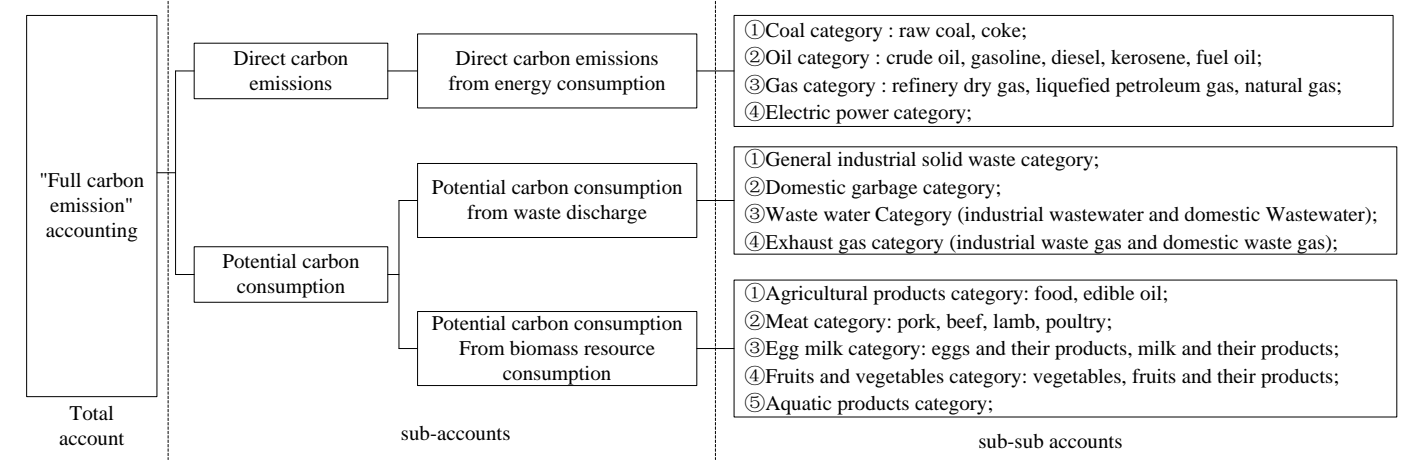

Figure 2. The framework of the full carbon accounting account 
According to Figure 2, the "full carbon emission" accounting is performed. Among them, the traditional energy consumption carbon emission conversion coefficient method (Wang et al., 2018) is applied in the calculation of the direct $\mathrm{CO}_{2}$ emission caused by energy consumption, while the potential $\mathrm{CO}_{2}$ emissions caused by waste discharge and biomass resource consumption are converted by emergy value conversion and its corresponding with $\mathrm{CO}_{2}$ emissions. The specific formula is as follows:

$$
\begin{aligned}
& T_{C O_{2}}=T_{C O_{2 e}}+T_{C O_{2 w}}+T_{C O_{2 b}}=\sum_{k=1}^{11} C O_{2 c k}+\sum_{n=1}^{4} C O_{2 w n}+\sum_{m=1}^{11} C O_{2 b m} \\
& =\sum_{k=1}^{11}\left(V_{k} \cdot \lambda_{k}\right)+\sum_{n=1}^{4} \frac{P_{n} \cdot \alpha_{n} \cdot \beta_{n}}{\gamma} \cdot \frac{0.8856}{3600000}+\sum_{m=1}^{11} \frac{U_{m} \cdot \alpha_{m} \cdot \beta_{m}}{\gamma} \cdot \frac{0.8856}{3600000}
\end{aligned}
$$

where: $T_{\mathrm{CO}_{2}}$ is the full $\mathrm{CO}_{2}$ emission of a certain city (full carbon emission); $T_{\mathrm{CO}_{\mathrm{ze}}}$ is direct carbon emissions caused by energy consumption; $T_{\mathrm{CO}_{2 w}}$ is potential carbon consumption caused by waste discharge; $T_{\mathrm{CO}_{2 b}}$ is potential carbon consumption caused by biomass resource consumption; $k$ is different categories of energy consumption for direct carbon emissions (such as raw coal, coke, crude oil, gasoline, diesel, kerosene, fuel oil, refinery dry gas, liquefied petroleum gas, natural gas and electricity); $\mathrm{CO}_{2 e k}$ is the direct $\mathrm{CO}_{2}$ emissions consumed in category $k ; n$ is different categories of waste emissions (such as industrial solid waste, domestic garbage, industrial wastewater, domestic wastewater, industrial waste gas and domestic waste gas) which will causing potential $\mathrm{CO}_{2}$ emissions; $\mathrm{CO}_{2 w n}$ is potential $\mathrm{CO}_{2}$ emissions from category $n$ waste; $b$ is biomass resource consumption which will causes potential $\mathrm{CO}_{2}$ emissions; $\mathrm{CO}_{2 b m}$ is the potential $\mathrm{CO}_{2}$ emissions caused by the $m$ type biomass resources consumption; $V_{k}$ is the raw data of the $k$ type energy consumption; $\lambda_{k}$ is the $\mathrm{CO}_{2}$ emission factor of the $k$ energy consumption; $P_{n}$ is the raw data of the $n$ type waste discharge; $\alpha_{n}$ is the $n$ type emergy conversion factor of waste; $\beta_{n}$ is emergy conversion rate of the $n$ type of waste; $U_{m}$ is the raw data of the $m$ type biomass resource; $\alpha_{m}$ is the emergy conversion coefficient of the $m$ type biomass resource, and $\beta_{m}$ is the emergy conversion rate of the $m$ type biomass resources; $\gamma$ is the emergy conversion rate of electric energy $(1.05 \times 105 \mathrm{sej} / \mathrm{J}), 1 \mathrm{kWh}=3600000 \mathrm{~J}$, and the power supply per kilowatt hour is $0.8856 \mathrm{~kg} \mathrm{CO}_{2}$ (Odum et al., 1955).

\section{Construction of SD model}

The SD model of JJJ Urban Agglomeration economic environmental complex system account is set up cored in KFs. The relationships of the factors in the target system are shown in Figure 3.

Variables representing flows of information and initials, arising as results of system activities and producing the related consequences are named as level variables which described as $\square$ in the flow diagram, while the rate variables are described as $\bar{z}$. Auxiliary variable means the detailed steps by which information associated with current levels are transformed into rates to bring about future changes. In addition, the symbol $\circlearrowleft$ represents the sinks or sources.

In Figure 3, there are four main level variables: total population of the city, annual GDP output, total energy consumption, total water use (because JJJ Urban Agglomeration is located in water scarce areas, so the indicator is selected). In addition, there is an important system output variable-total carbon emission. All the four main 
level variables and the one important output variable is described as $\square$. The other variables follow the expression principle of system dynamics flow chart.

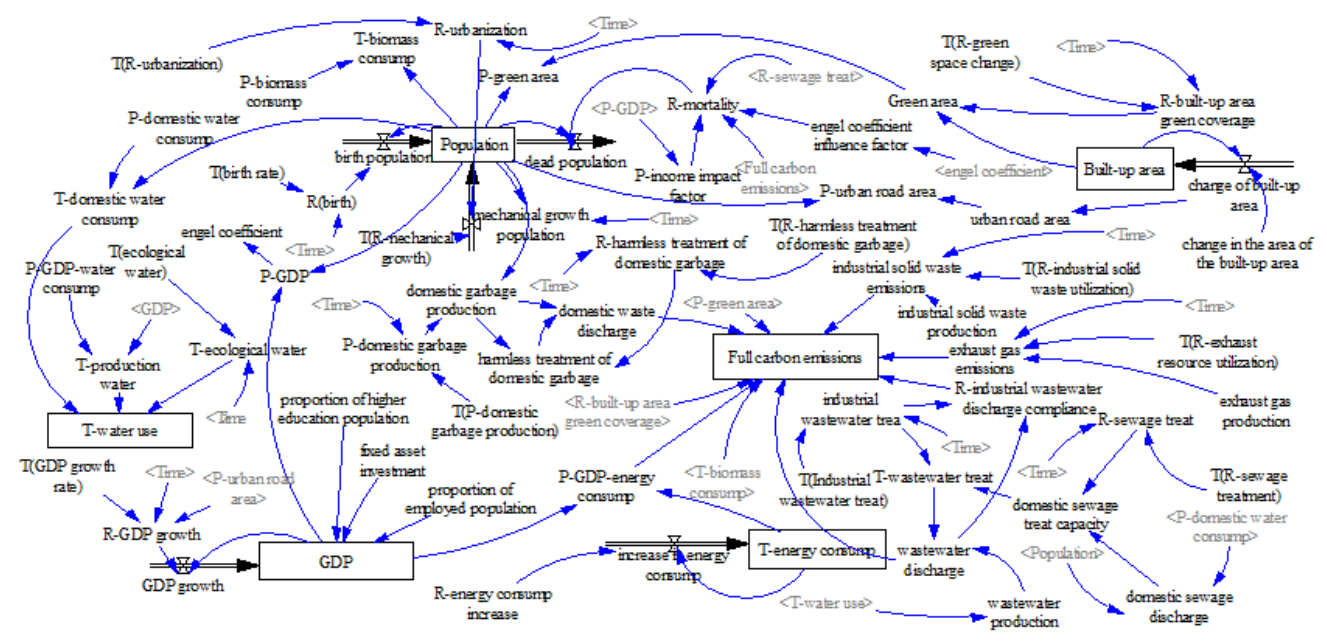

Figure 3. the flow chart of JJJ Urban Agglomeration

The SD model takes certain steps along the time axis in the simulation process. At the end of each step, the system variables denoting the state of the system are updated to represent the consequences resulting from the previous simulation step (Zhang et al., 2008). Initial conditions are needed in the first time step. According Figure 3 and the above principle, we can compile the system dynamics equations of each node city of JJJ Urban Agglomeration one by one based on collecting, collating and analyzing the historical data of the system.

After SD model construction, it is necessary to identify whether the model can be in line with the real situations by the validity test and sensitivity analysis. Accordingly, parameters and relevance can be modified and confirmed until the SD model has good robustness and can reflect the real system in different scenarios.

\section{Construction low-carbon economic linkages strength model}

Based on the gravitational model, this paper combines the traditional economic linkages strength model with the "full carbon emission" model to construct the lowcarbon economic linkages strength model as follows:

$$
R_{i j}=\frac{\sqrt[3]{\frac{P_{i}}{T_{C O_{2 i}}} \cdot \frac{G_{i}}{T_{C O_{2 i}}} \cdot \frac{S_{i}}{T_{C O_{2 i}}}}}{D_{i j}} \cdot \frac{\sqrt[3]{\frac{P_{j}}{T_{C O_{2 j}}} \cdot \frac{G_{j}}{T_{C O_{2 j}}} \cdot \frac{S_{j}}{T_{C O_{2 j}}}}}{D_{i j}} \cdot M_{1} \cdot M_{2} \cdot M_{3}
$$

where: $i$ and $j$ are the central city and the irradiated city and $R_{i j}$ is the low-carbon economic linkages strength of the central city $i$ accepted by the radiated city $j ; P_{i}$ and $P_{j}$ are the population size of the $i$ and $j$ cities respectively; $G_{i}$ and $G_{j}$ are the regional gross domestic product (GDP) of cities $i$ and $j$ respectively; $S_{i}$ and $S_{j}$ are the road areas of cities $i$ and $j$ respectively; $T_{C O_{n i}}$ and $T_{C_{2 j}}$ are cities of $i$ and $j$ annual $\mathrm{CO}_{2}$ emissions respectively; $D_{i j}$ is the distance between cities $i$ and $j ; M_{l}$ is the human resources adjustment coefficient, 
which is affected by factors such as the proportion of urban employment density, the proportion of people receiving higher education (the proportion of the number of undergraduates who are over 6 years old in the permanent population of the city), the influence of natural growth rate and urbanization level; $M_{2}$ is the economic resource adjustment coefficient, which is affected by factors such as per capita GDP, economic density and per capita fixed assets investment; $M_{3}$ is the natural resource adjustment coefficient, which is affected by factors such as the population per square kilometer, per capita domestic water consumption and urban per capita green area.

According to the principle of pressure superposition, the strength of economic connection from a single source centered on a single city is calculated, and the source superposition formula is used to superpose the low-carbon economic linkages strength of two-source (two central cities) or three-source (three central cities). The formula is as follows:

$$
R_{j}=10 \cdot \lg \left(10^{\wedge 0.1 R_{i j} j}+10^{\wedge 0.1 R_{i_{2 j}}}+10^{\wedge .1 R_{i, j}}\right)
$$

where: $R_{j}$ is the low-carbon economic linkages strength of the radiated city $j$ after accepting the superposition of two or three sources of radiation; $R_{i_{1} j}, R_{i_{2} j}$ and $R_{i_{3} j}$ are different central cities $i_{1}, i_{2}$ and $i_{3}$ radiate the low-carbon economic linkages strength radiation of the same radiated city $j\left(R_{i_{3} j}\right.$ is not included when the two sources are superimposed).

After calculation of the low-carbon economic linkages strength, the distribution of social economic resources and environment in urban agglomerations can be judged by fractal dimension. The fractal dimension can be calculated as:

$$
K \propto R_{(\mathrm{K})}^{-D}
$$

where: $K$ is the number of cities in the region; $R$ is the intensity of low-carbon economic linkages strength between cities; $D$ is the fractal dimension. Take the logarithm of the two sides of Equation 3, we can get:

$$
\ln R_{(k)}=A-\frac{1}{D} \ln k
$$

where: $k$ is the number of cities (counties) low-carbon economic connection gravity ranking sequences, and $A$ is an equal constant.

The basic criteria for judging rationality are as follows:

When $D=1$, the urban agglomeration is a constrained order-scale distribution, which means that the radiation received by cities in urban agglomerations from central cities is an absolute average, not a gradient circle. It is impossible to form a high-to-low order transmission and radiation of the internal energy of the urban agglomeration (low-carbon economic linkages strength) and the radiation efficiency is not optimal.

When $D>1$, the central city in the urban agglomeration has lost the advantage of high gradient, which is not conducive to its overall development of the urban agglomeration.

When $D<1$, there is a difference in the distribution of economic linkages strength. From the above analysis, the best fractal dimension is $D<1$. 
Li et al. (1992) used the golden section principle and the Fibonacci sequence to derive a judgment that can be used as a reasonable distribution of the network system. That is the golden section 0.618 as the reasonable fractal dimension judgment point. According this judgment, when $D=0.618$, the urban agglomeration is in the optimal connection gravity of the urban agglomeration.

\section{Results}

\section{Data sources}

The data of the scenarios analysis are derived from the 2007-2018 China Statistical Yearbook and the statistical yearbooks of relevant cities, the China Energy Statistical Yearbook and the relevant cities' energy statistics yearbooks and the China Environmental Statistics Yearbook. Meanwhile, some of the data are derived from the iFind. The data in this paper covers the economic development status, social development status, environmental quality and resource utilization of cities in the study.

\section{Scenarios design}

In this paper, five scenarios are carried out to analyze the strength and dimension of urban low-carbon economic linkages for analyzing the efficiency of JJJ collaborative development under different low carbon economy strategies around Xiongan. And the scenarios designed of this paper is based on the following basic hypothesis: (1) The development of JJJ Urban Agglomeration follows the relevant national and local planning, which does not cover areas and relevant indicators follow the current development trend (based on the prediction of SD model); (2) In the current scenario, Xiongan has the same economic density, population density and carbon emissions intensity as Beijing.

The five scenarios are designed as follows:

Scenario 1: "Beijing-Tianjin" dual source radiation under inertial scenarios. This paper selects the data from 2006 to 2017 as the historical data of the JJJ Urban Agglomeration. Based on the JJJ Urban Agglomeration eco-economic development system dynamics model (Wang et al., 2013), it predicts the carbon emissions and related indicators of cities in the JJJ Urban Agglomeration from 2018 to 2030.

Scenario 2: "Beijing-Tianjin-Xiongan" three-source radiation under inertia scenario. The operational data is the same as scenario 1, except that Xiongan is listed as a radiation source.

Scenario 3: Xiongan achieves carbon peaks ahead of schedule in 2018, and after reaching the peak, carbon emissions are reduced by $0.5 \%$ per year on the basis of scenario 1. Meanwhile the development trends and carbon emissions of other cities remain unchanged.

Scenario 4: On the basis of Scenario 3, Beijing and Tianjin are also used as radiation sources, and the development trend remains unchanged. The amount of carbon emissions is reduced by $0.5 \%$ per year on the basis of Scenario 1, and other cities remain unchanged.

Scenario 5: Based on Scenario 4, the development trend of other cities remains unchanged, and carbon emissions are reduced by $0.5 \%$ per year on the basis of Scenario 1. 


\section{Results analysis}

Based on the above five scenarios, this part applied Equation 1 to calculate the total carbon emissions, Equation 2 to calculate the low-carbon economic linkages strength of single-center sources, Equation 3 to superpose the low-carbon economic linkages strength of multi-center sources, and Equations 4 and 5 to calculate the fractal dimension value. The calculation results are shown in Figure 4.

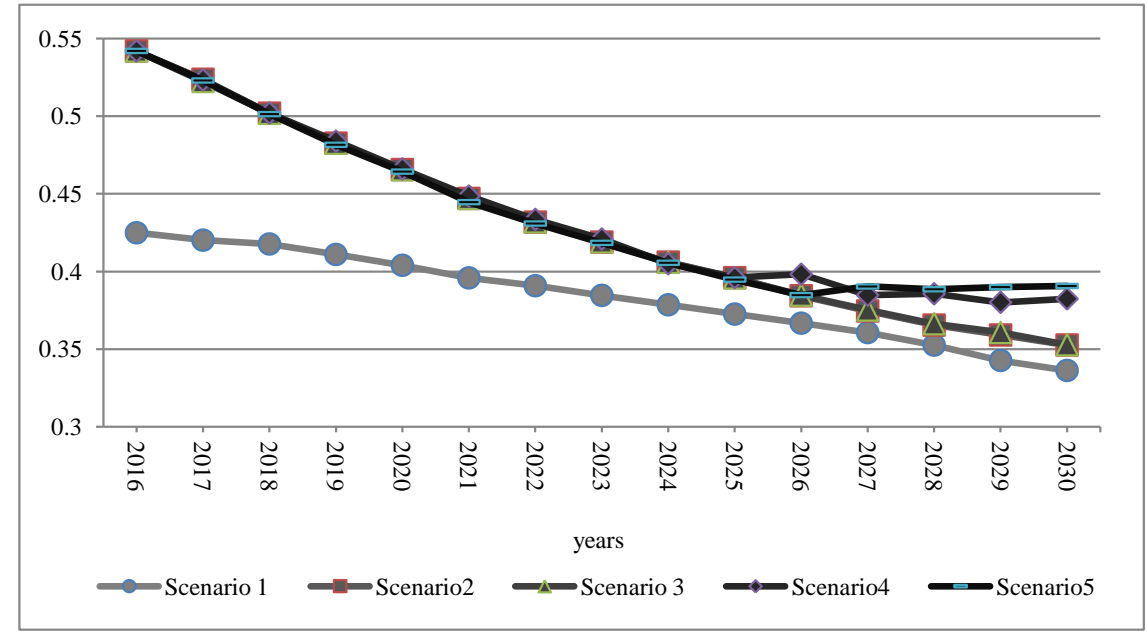

Figure 4. 2016-2030 JJJ Urban Agglomeration low-carbon economic linkages strength fractal dimension

Building Xiongan as a radiation center of JJJ multi-center urban agglomeration can significantly promote the collaborative development of urban agglomeration

It can be seen from Figure 4 that from 2016 to 2030, the fractal dimension of the low-carbon economic linkages strength of Scenario 2 is generally higher than the fractal dimension of Scenario 1, which indicates that "Beijing-Tianjin-Xiongan" as the radiation center is better than the "Beijing-Tianjin" dual source as the radiation center.

Synchronized upgrading of multicenter source level can better promote the development of JJJ Urban Agglomeration

From 2016 to 2025, the low-carbon economic linkages strength fractal dimension from Scenario 3 to Scenario 5 is basically equal. By 2026, there will be a divergence except for individual years, the fractal dimension of scenario 4 and scenario 5 increased steadily, while the fractal dimension of scenario 3 decreased steadily. The above comparison results show that improving the low-carbon level of Xiongan alone could not influence on the low-carbon development of JJJ Urban Agglomeration, while the effect is significant when Beijing, Tianjin and Xiongan low-carbon level is improved at the same time.

The delay of radiation from high-gradient to low-gradient will not affect the low-carbon coordinated development of JJJ Urban Agglomeration

There will be a delay in the transmission of low-carbon economic development capacity from high to low gradient. Figure 4 shows that from 2016 to 2028, the fractal 
dimension values of scenario 4 and scenario 5 were basically same. Until 2029, scenario 4 and scenario 5 developed differently. The fractal dimension of scenario 5 began to be higher than scenario 4. The above comparison shows that the delay of low-carbon development capability radiation will not affect the radiation effect of high gradient cities (central source cities) on low gradient cities, nor does it affect the coordinated development of urban agglomerations.

\section{Discussion and conclusions}

\section{Discussion}

There two points needed to be discussed:

(1) Based on the systematic analysis of the city, this paper studies the effect of Xiongan on the coordinated development of JJJ Urban Agglomeration under different scenarios from the perspective of low-carbon economy. In this paper, the source superposition method is used to measure the low-carbon economic linkages between cities in urban agglomeration under the dual-source and triple-source scenarios. This method is more realistic in describing the radiation situation of the central city to the surrounding cities in space. It has universality and generalization, and can be applied to different urban agglomerations. The difference from this paper is that you need to adjust the source superposition formula according to the number of radiation sources in urban agglomerations.

(2) In order to analyze the radiation effects of low-carbon economy under different carbon emission conditions, five schemes are established according to different total carbon emissions. Since scenario 1 and scenario 2 have the same total carbon emissions, we only choose scenarios 2, 3, 4 and 5 for analysis. The full carbon emissions of JJJ Urban Agglomeration under scenarios 2, 3, 4 and 5 are shown in Table 1.

Table 1. Full carbon emissions of JJJ Urban Agglomeration under different scenarios

\begin{tabular}{l|c|c|c|c}
\hline Years & Scenario 2 & Scenario 3 & Scenario 4 & Scenario 5 \\
\hline 2016 & 17.5603 & 17.5603 & 17.5426 & 17.5426 \\
2017 & 16.4905 & 16.4887 & 16.4562 & 16.4081 \\
2018 & 16.4260 & 16.4241 & 16.3912 & 16.3438 \\
2018 & 16.4795 & 16.4738 & 16.4400 & 16.3931 \\
2020 & 16.6840 & 16.6735 & 16.6389 & 16.5920 \\
2021 & 16.8278 & 16.8260 & 16.7914 & 16.7437 \\
2022 & 17.0700 & 17.0683 & 17.0335 & 16.9847 \\
2023 & 17.4918 & 17.4901 & 17.4548 & 17.4043 \\
2024 & 17.9335 & 17.9319 & 17.8963 & 17.8438 \\
2025 & 18.5864 & 18.5848 & 18.5485 & 18.4935 \\
2026 & 19.0421 & 19.0406 & 19.0051 & 18.9469 \\
2027 & 19.6471 & 19.6456 & 19.6110 & 19.5489 \\
2028 & 20.4149 & 20.4135 & 20.3798 & 20.3129 \\
2029 & 21.0656 & 21.0642 & 21.0317 & 20.9602 \\
2030 & 22.1694 & 22.1681 & 22.1369 & 22.0586 \\
\hline
\end{tabular}


As can be seen from Table 1, Scenario 5 has the strongest carbon reduction compared with Scenario 4, but the increase of fractal dimension is not obvious. On the contrary, scenario 4 has less carbon reduction than scenario 5, but its fractal dimension has been greatly improved. The greater the carbon reduction is, the higher the cost to be borne. Therefore, scenario 5 is much higher than scenario 4 in terms of carbon emission reduction costs, but the radiation effect has not been greatly improved. In summary, there is the most obvious carbon reduction effect in Scenario 4. So if the central cities reduce carbon in advance and grasp the key points of urban agglomeration development, they can achieve better results with less investment, which also validates conclusion 3.

\section{Conclusions}

The above fractal dimension analysis of of JJJ Urban Agglomeration show:

(1) As one radiation center of JJJ Urban Agglomeration, Xiongan can significantly promote the collaborative development of the area;

(2) For the development of the whole area, the effect of improving the low-carbon level of Xiongan alone is much less than improving the low-carbon level of Beijing, Tianjin and Xiongan at the same time;

(3) The delay which comes from the transmission from high to low gradient will not affect the radiation on the coordinated development of urban agglomerations.

In the process of low-carbon development and construction of JJJ Urban Agglomeration, it is necessary to pay attention to Xiongan's construction as a highgradient radiation center, and improve the low-carbon level of all radiation centers in the urban agglomeration at the same time. In the near future, the low-carbon coordinated development of JJJ Urban Agglomeration will be realized at the lowest possible cost.

Acknowledgements. The authors acknowledge the National Social Science Fund of China (18BJY079) and Tianjin Philosophy and Social Science Planning Project (TJLJ15-008).

\section{REFERENCES}

[1] Albeverio, S., et al. (2008): The Dynamics of Complex Urban Systems. - Physica-Verlag, Heidelberg.

[2] Camagni, R., Capello, R., Nijkamp, P. (1998): Towards sustainable city policy: an economy-environment technology nexus. - Ecological Economics 24(1): 103-118.

[3] Cao, Y., Wei, L., Yu, H. (2012): Optimization study of provincial urban agglomeration logistics alliance system based on the SD model. - Journal of Chongqing Jiaotong University 30(2): 334-339.

[4] Duranton, G., Puga, D. (2003): Micro-foundations of urban agglomeration economies. Social Science Electronic Publishing 4(4): 2063-2117.

[5] Du, L., Li, X., Zhao, H., et al. (2018): System dynamic modeling of urban carbon emissions based on the regional national economy and social development plan: a case study of Shanghai city. - Journal of Cleaner Production 172: 1501-1513.

[6] Elliot, T., Rugani, B., BabíAlmenar, J., et al. (2018): A proposal to integrate system dynamics and carbon metabolism for urban planning. - Procedia Cirp. 69: 78-82.

[7] Frankhauser, P. (1998): European cities - insights on outskirts. - Report Cost Action 10: 79-105. 
[8] Jia, P., Li, K., Shao, S. (2018): Choice of technological change for China's low-carbon development: evidence from three urban agglomerations. - Journal of Environmental Management 206: 1308-1319.

[9] Jefferson, J., B. (2013): Dark side of the planet: hidden dimensions of urban agglomeration. - Urban Geography 39(10): 1-8.

[10] Kang, Z. Y., Li, K., Qu, J. (2018): The path of technological progress for China's lowcarbon development: evidence from three urban agglomerations. - Journal of Cleaner Production 178: 644-654.

[11] Li, X., Wang, L., Ji, D., et al. (2013): Characterization of the size-segregated watersoluble inorganic ions in the Jing-Jin-Ji urban agglomeration: Spatial/temporal variability, size distribution and sources. - Atmospheric Environment 77(7): 250-259.

[12] Lu, S., Minoru, F., Tomohiro, T., et al. (2018): Improving waste to energy rate by promoting an integrated municipal solid-waste management system. - Resources, Conservation and Recycling 136: 289-296.

[13] Li, H., Ai, N. (1992): Market network with golden section features and fractal properties. - Journal of Economic Geography (4): 1-5.

[14] Odum, H. T., Odum, E. P. (1955): Trophic structure and productivity of a windward coral reef community on Eniwetok Atoll. - Ecological Monographs 25(3): 291-320.

[15] Pan, X., Zhang, Y., Xu, L., et al. (2009): An analysis of farmers' perception of the new cooperative medical system in Liaoning Province, China. - BMC Health Services Research 9(1): 1-8.

[16] Rich, K. M., Rich, M., Dizyee, K. (2016): Participatory systems approaches for urban and peri-urban agriculture planning: the role of system dynamics and spatial group model building. - Agricultural Systems 160: 110-123.

[17] Tan, R. R., Aviso, K. T., Foo, D. C. Y. (2018): Carbon emissions pinch analysis of economic systems. - Journal of Cleaner Production 182: 863-871.

[18] Tannier, C., Thomas, I. (2013): Defining and characterizing urban boundaries: a fractal analysis of theoretical cities and Belgian cities. - Computers, Environment and Urban Systems 41: 234-248.

[19] Tannier, C., Thomas, I., Vuidel, G., et al. (2011): A fractal approach to identifying urban boundaries. - Geographical Analysis 43(2): 211-227.

[20] Timothy, D., Searchinger, S., Wirsenius, T., Beringer, P. D. (2018): Assessing the efficiency of changes in land use for mitigating climate change. - Nature 564(7735): 249264.

[21] Wu, D., Ning, S. (2018): Dynamic assessment of urban economy-environment-energy system using system dynamics model: a case study in Beijing. - Environmental Research 164(7): 70-84.

[22] White, R. (1994): Urban systems dynamics and cellular automata: fractal structures between order and chaos. - Chaos, Solitons \& Fractals 4(4): 563-583.

[23] Wang, S., Su, Y., Zhao, Y. (2018): Regional inequality, spatial spillover effects and influencing factors of China's city-level energy-related carbon emissions. - Journal of Geographical Sciences 73(3): 414-428.

[24] Wang, X. (2013): Research on Regional Ecological Economic Evaluation Based on System Dynamics Method. - Tianjin Polytechnic University, Xiqing.

[25] Yu, Y., Wen, Z. (2010): Evaluating China's urban environmental sustainability with data envelopment analysis. - Ecological Economics 69(9): 1748-1755.

[26] Yu, Y., He, J., Tang, W., et al. (2018): Modeling urban collaborative growth dynamics using a multiscale simulation model for the Wuhan urban agglomeration Area, China. ISPRS International Journal of Geo-Information 7(5): 176-188.

[27] Zhou, D., Bonafoni, S., Zhang, L., et al. (2018): Remote sensing of the urban heat island effect in a highly populated urban agglomeration area in east China. - Science of the Total Environment 628: 415-429. 
[28] Zhang, X. H., Zhang, H. W., Chen, B., et al. (2008): Water resources planning based on complex system dynamics: a case study of Tianjin city. - Communications in Nonlinear Science and Numerical Simulation 13(10): 2328-2336.

[29] Zhang, X., Li, X., Ye, W., Zhang, B. (2015): Calculation of full carbon emission and evaluation of carbon emission performance. - Acta Scientiarum Naturalium Universitatis Pekinensis 51(4): 639-646. 